\title{
Amortizing Garbled Circuits
}

\author{
Yan Huang ${ }^{1}$, Jonathan Katz ${ }^{1}$, Vladimir Kolesnikov ${ }^{2}$, \\ Ranjit Kumaresan ${ }^{3}$, and Alex J. Malozemoff ${ }^{1}$ \\ 1 University of Maryland, College Park, MD, USA \\ \{yhuang, jkatz, amaloz\}@cs.umd.edu \\ ${ }_{2}$ Bell Labs, Murray Hill, NJ, USA \\ kolesnikov@research.bell-labs.com \\ 3 Technion, Haifa, Israel \\ ranjit@cs.technion.ac.il
}

\begin{abstract}
We consider secure two-party computation in a multipleexecution setting, where two parties wish to securely evaluate the same circuit multiple times. We design efficient garbled-circuit-based two-party protocols secure against malicious adversaries. Recent works by Lindell (Crypto 2013) and Huang-Katz-Evans (Crypto 2013) have obtained optimal complexity for cut-and-choose performed over garbled circuits in the single execution setting. We show that it is possible to obtain much lower amortized overhead for cut-and-choose in the multiple-execution setting.

Our efficiency improvements result from a novel way to combine a recent technique of Lindell (Crypto 2013) with LEGO-based cut-andchoose techniques (TCC 2009, Eurocrypt 2013). In concrete terms, for 40 -bit statistical security we obtain a $2 \times$ improvement (per execution) in communication and computation for as few as 7 executions, and require only 8 garbled circuits (i.e., a $5 \times$ improvement) per execution for as low as 3500 executions. Our results suggest the exciting possibility that secure two-party computation in the malicious setting can be less than an order of magnitude more expensive than in the semi-honest setting.
\end{abstract}

\section{Introduction}

Two-party secure computation $(2 \mathrm{PC})$ is a rapidly developing area of cryptography. While the basic approach for semi-honest security, garbled circuits (GC) [27], is extensively studied and is largely settled, security against malicious players has seen recent significant improvements. The classical technique for lifting the GC approach to work in the malicious setting is cut-and-choose $(\mathrm{C} \& \mathrm{C})$, formalized and proven secure by Lindell and Pinkas [15. Until recently, this approach required significant overhead: to guarantee probability of cheating $<2^{-s}$, approximately $3 s$ garbled circuits needed to be generated and sent. However, in Crypto 2013 two works reduced the number of garbled circuits required in cut-and-choose to $s+O(\log s)[9]$ and to $s[14$.

Our Contribution. We further significantly reduce the replication factor for C\&C-based protocols in the multiple execution setting, where the same function 
(possibly with different inputs) is evaluated multiple times either in parallel or sequentially. To achieve this, we combine in a novel way the "fast C\&C" technique of Lindell [14] with the "LEGO C\&C" technique [6]22].

Our Setting and Motivation. We consider the multiple execution setting, where two parties compute the same function on possibly different inputs either in parallel or sequentially. Here we argue that multiple evaluations of the same function is indeed a natural and frequently-occurring important scenario.

Today, $2 \mathrm{PC}$ is only beginning to enter practical deployment. However, we can reasonably speculate on likely future use cases. In the commercial setting, $2 \mathrm{PC}$ is natural in both business-to-business and business-to-customer interactions. For example, a bank customer could perform financial transactions (e.g., payments or transfers), a cell phone customer could perform private location-based queries, two businesses or government agencies might query their joint databases of customers, etc. In all of these scenarios, many of the securely evaluated functions are the same, only differing on their inputs. In fact, we conjecture that singleexecution functions may be less likely to be used in commercial settings. This is because, as a rule-of-thumb of security, externally-accessible interfaces need to be clean and standardized. Allowing a small number of predetermined customer actions allows for more manageable overall security.

Additionally, many complex protocols from the research literature include multiple executions of the same function evaluated on different inputs. For example, Gordon et al. [8] propose sublinear 2PC based on oblivious RAM (ORAM). In their protocol, each ORAM step is executed by evaluating the same function using 2PC. Another frequently used subroutine is oblivious PRF, used, e.g., in the previously mentioned sublinear $2 \mathrm{PC}$ work 8 as well as in private database searches [12]. A recent such work 23. traverses the database search tree by evaluating the same match function at each tree node. Finally, any two universal circuits (of the same size) are implementing the same function.

\subsection{Preliminaries}

Let $s$ denote the statistical security parameter; namely, an adversary can succeed in cheating with probability up to $2^{-s}$. Let $n$ denote the computational security parameter. We let $t$ denote the total number of times the parties wish to evaluate a given circuit, and let $\rho=\rho(s, t)$ represent the number of circuits, per evaluation, that need to be generated to achieve an error probability of $2^{-s}$. Before discussing our specific technical contribution, we recall the main ideas of our building blocks.

Fast Cut-and-Choose Using Cheating Punishment [14]. Cut-and-choose (C\&C) protocols for GCs work by letting circuit constructor $P_{1}$ generate and send a number of GCs to the evaluator $P_{2}$, who then chooses a subset of circuits to open and check for correctness. If the checks pass, the remaining circuits are evaluated as in Yao's protocol [27, and the final output is obtained by taking majority over the individual outputs. In concrete terms, prior works [15 25] required at least 125 circuits to be sent by $P_{1}$ to guarantee security $2^{-40}$. Lindell's 
improved technique 14 achieves $2^{-s}$ security while requiring $P_{1}$ to send only $s$ circuits (i.e., 40 circuits for $2^{-40}$ security).

Lindell's protocol (which we call the "fast C\&C" protocol) has two phases. In the first phase, $P_{1}$ with input $x$ and $P_{2}$ with input $y$ run a modified $\mathrm{C} \& \mathrm{C}$ which ensures that $P_{2}$ obtains a proof of cheating $\phi$ if it receives two inconsistent output values in any two evaluation circuits. Now, if all evaluation circuits produce the same output $z, P_{2}$ locally stores $z$ as its output. Both parties always continue to the second cheating-punishment phase. In it, $P_{1}$ and $P_{2}$ securely evaluate a smaller circuit $C^{\prime}$, which takes as inputs $P_{1}$ 's input $x$ and $P_{2}$ 's proof $\phi .\left(P_{2}\right.$ inputs random values if he does not have $\phi$.) $P_{1}$ proves in zero-knowledge the consistency of its input $x$ between the two phases. $C^{\prime}$ outputs $x$ to $P_{2}$ if $\phi$ is a valid proof of cheating; otherwise $P_{2}$ receives nothing. The efficiency improvement is due to the fact that cheating is punished if there is any inconsistency in outputs.

LEGO Cut-and-Choose [6,22]. These works take a different approach by implementing a two-stage $\mathrm{C} \& \mathrm{C}$ at the gate level. The evaluation circuit is then constructed from the unopened garbled gates. In the first stage, $P_{1}$ sends multiple garbled gates and $P_{2}$ performs a standard C\&C with replication factor $\rho(s)=$ $O(s / \log |C|) . P_{2}$ aborts if any opened gate is garbled incorrectly. In the next stage, $P_{2}$ partitions the $\rho(s)|C|$ garbled gates into buckets such that each bucket contains $O(\rho(s))$ garbled gates. This two-stage C\&C ensures that, except with probability $2^{-s}$, each bucket contains a majority of correctly constructed garbled gates.

To connect gates with one another, Nielsen and Orlandi 22 use homomorphic Pedersen commitments. The resulting computational efficiency is relatively poor as they perform several expensive public-key operations per gate. This is addressed in the miniLEGO work [6], where the authors (among other things) construct homomorphic commitments from oblivious transfer (OT), whose cost can be amortized by OT extension [10. However, the overall efficiency of this construction is still lacking in concrete terms due to large constants inside the big-O notation. In particular, the communication efficiency is adversely affected by the use of asymptotically constant-rate codes that are concretely inefficient.

\subsection{Overview of Our Approach}

Our main idea for the multiple execution setting is to run two-stage LEGO C\&C at the circuit level, and then use fast $\mathrm{C} \& \mathrm{C}$ in the second stage (thereby requiring only a single correctly constructed circuit from each bucket). In particular, now the size of $C^{\prime}$ used in each execution depends only on the input and output lengths of $C$, and is no longer proportional to $|C|$. In this section, we focus only on the cut-and-choose aspect of the protocol; namely, on preventing $P_{1}$ 's cheating by submitting incorrect garbled circuits. More detailed protocol descriptions for both the parallel and sequential settings can be found in Section 2 and Section 3

In the first-stage cut-and-choose, $P_{1}$ constructs and sends to $P_{2}$ a total of $\rho t$ GCs. Next, $P_{2}$ requests that $P_{1}$ open a random $\rho t / 2$-sized subset of the garbled circuits. If $P_{2}$ discovers that any opened garbled circuit is incorrectly constructed, 
Table 1. The number of garbled circuits required per execution in order to guarantee a security loss of $<2^{-40}$. For comparison, the last two columns show the number of circuits required by the fast $\mathrm{C} \& \mathrm{C}$ protocol 14 in the parallel and sequential settings. Note that when using the fast $\mathrm{C} \& \mathrm{C}$ protocol for sequential executions we need to increase the replication factor from $s$ to $s+\log t$.

\begin{tabular}{cccc}
\hline \# of Executions & $\begin{array}{c}\text { Replication } \\
\text { parallel/sequential }\end{array}$ & \multicolumn{2}{c}{ Replication for Fast C\&C } \\
parallel & sequential \\
\hline 2 & 32 & 40 & 41 \\
4 & 24 & 40 & 42 \\
7 & 20 & 40 & 42 \\
20 & 16 & 40 & 44 \\
100 & 12 & 40 & 46 \\
3500 & 8 & 40 & 51 \\
\hline
\end{tabular}

it aborts. Otherwise, $P_{2}$ proceeds to the second stage cut-and-choose, where it randomly assigns unopened circuits to $t$ buckets such that each bucket contains $\rho / 2$ circuits. Now, as in the fast C\&C protocol [14, each of the $t$ evaluations are executed in two phases. In the first phase of the $k$ th execution, party $P_{2}$ evaluates the $\rho / 2$ evaluation circuits contained in the $k$ th bucket. The circuits are designed such that if $P_{2}$ obtains different outputs from evaluating circuits in the $k$ th bucket, then it obtains a proof of cheating $\phi_{k}$. Next, both parties continue to the cheating-punishment phase, where $P_{1}$ and $P_{2}$ securely evaluate a smaller circuit that outputs $P_{1}$ 's input $x_{k}$ if $P_{2}$ provides a valid proof $\phi_{k}$.

Clearly, $P_{1}$ succeeds in cheating only if (1) it constructed $m \geq \rho / 2$ bad circuits, (2) none of these $m$ bad circuits were caught in the first cut-and-choose stage (i.e., $m \leq \rho t / 2$ ), and (3) in the second stage, there exists a bucket that contains all bad circuits. It is easy to see that the probability with which $m$ bad circuits escape detection in the first stage cut-and-choose is $\left(\begin{array}{c}\rho t-m \\ \rho t / 2\end{array}\right) /\left(\begin{array}{c}\rho t \\ \rho t / 2\end{array}\right)$. Conditioned on this event happening, the probability that a particular bucket contains all bad circuits is $\left(\begin{array}{c}m \\ \rho / 2\end{array}\right) /\left(\begin{array}{c}\rho t / 2 \\ \rho / 2\end{array}\right)$. Applying the union bound, we conclude that the probability that $P_{1}$ succeeds in cheating is bounded by

$$
t\left(\begin{array}{c}
\rho t-m \\
\rho t / 2
\end{array}\right)\left(\begin{array}{c}
m \\
\rho / 2
\end{array}\right) /\left(\begin{array}{c}
\rho t \\
\rho t / 2
\end{array}\right)\left(\begin{array}{c}
\rho t / 2 \\
\rho / 2
\end{array}\right) .
$$

For any given $t$ and $s$, the smallest $\rho$, hinging on the maximal probability of $P_{1}$ 's successful attack, can be determined by enumerating over all possible values of $m$ (i.e., $\{\rho / 2, \rho / 2+1, \ldots, \rho t / 2\})$.

As an example, for $t=20$ in a parallel execution setting with $s=40$, using our protocol the circuit generator needs to construct $16 \cdot t=320$ garbled circuits, whereas using a naïve application of Lindell's protocol [14] requires $40 \cdot t=800$ garbled circuits.

Parallel vs. Sequential Executions. As will be evident, it is important to distinguish between the settings where multiple evaluations are carried out in parallel (e.g., when all inputs are available at the start of the protocol) and 
where these evaluations are carried out sequentially (e.g., when not all inputs are available as they, for example, depend on the outputs of previous executions). Below, we provide an overview of the main challenges of each setting, and an outline of our solutions.

Parallel executions. Under the DDH assumption, we apply our C\&C technique in the parallel execution setting by modifying Lindell's protocol [14 as follows. We construct a generalized cut-and-choose oblivious transfer (C\&C OT) functionality that supports multi-stage cut-and-choose. We call this functionality $\mathcal{F}_{\text {mcot }}$. Asymptotically, we can realize $\mathcal{F}_{\text {mcot }}$ using general secure computation, since the circuit for $\mathcal{F}_{\text {mcot }}$ depends only on the length of $P_{2}$ 's input and is otherwise independent of the circuit. However, such a realization is extremely inefficient in practice (the size of the circuit for realizing $\mathcal{F}_{\text {mcot }}$ needs to accept inputs of length at least $n \rho t \ell$, where $n$ is the computational security parameter and $\ell$ is the input length). Instead, we show an efficient realization that is only a factor $\rho t^{2} / s$ less efficient (per execution) than the modified C\&C OT realization of Lindell [14]. We elaborate more on this, and other important details, in Section 2

Sequential executions. To prevent a malicious evaluator from choosing its inputs based on the garbled circuit, GC-based 2PC protocols perform OT before the constructor sends its GCs to the evaluator (i.e., before the cut-and-choose phase). This forces the parties, and in particular the evaluator, to "commit" to their inputs before performing the cut-and-choose. This, however, does not work in the sequential setting, where the parties may not know all their inputs at the beginning of the protocol. Standard solutions used in previous works [1/7/20 include assuming the garbled-circuit construction is adaptively secure or using adaptively-secure garbling [3] explicitly, assuming the programmable randomoracle model. Another issue is that since now we perform OTs for each execution separately, we can no longer use C\&C OT or its variants; instead we rely on the "XOR-tree" approach of Lindell and Pinkas [15] to avoid selective failure attacks. We elaborate more on this, and other details, in Section 3 .

Our solution for the sequential setting readily carries over to the parallel setting. In particular, adapting our protocol from the sequential to the parallel setting may address situations where the cost incurred by the use of $\mathcal{F}_{\text {mcot }}$ outweighs the cost of using both the XOR-tree approach and adaptively secure garbled circuits.

\subsection{Related Work}

Lindell and Pinkas 15 gave the first 1 rigorous $2 \mathrm{PC}$ protocol based on cut-andchoose. For $s=40$, their protocol required at least $17 s=680$ garbled circuits. Subsequent work by the same authors [16] reduced the number of circuits to 128. This was later improved by shelat and Shen 25] to 125 using a more precise analysis of the C\&C approach. In Crypto 2013, two works 914 proposed (among other things) dramatic improvements to the number of garbled circuits that need

${ }^{1}$ C\&C mechanisms were previously employed in works by Pinkas [24] and Malkhi et al. 18 but these approaches were later shown to be flawed 1319. 
to be sent. In more detail, for achieving statistical security $2^{-s}$, Huang et al.'s protocol 9] requires $2 s+O(\log s)$ circuits, where each party generates half of them, and Lindell's protocol 14 requires exactly $s$ circuits.

While all of the above works perform cut-and-choose over circuits, applying cut-and-choose at the gate-level has also been considered [562122. As discussed above, this approach naturally extends to the multiple execution setting, and furthermore is not inherently limited to considering settings where the same function is evaluated multiple times. Nielsen et al. 21] indeed show concrete efficiency improvements using gate-level cut-and-choose techniques. However, the number of rounds grows linearly with the depth of the evaluated circuit.

Finally, in independent and concurrent work, Lindell and Riva 17] also investigate the multiple execution setting, and obtain performance improvements similar to ours. An interesting difference between our works is that while we always let the evaluator pick half the circuits to check, they show that varying the number of check circuits can lead to an additional performance improvement.

\section{The Parallel Execution Setting}

Consider a setting where two parties wish to securely evaluate the same function multiple times in parallel. Let $f$ denote the function of interest, and let $t$ denote the number of times the parties wish to evaluate $f$. Let $P_{1}$ 's (resp., $P_{2}$ 's) input in the $k$ th execution be $x_{k}$ (resp., $\left.y_{k}\right)$, and let $x=\left(x_{1}, \ldots, x_{t}\right)$ and $y=\left(y_{1}, \ldots, y_{t}\right)$. We define $f^{(t)}(x, y)=\left(f\left(x_{1}, y_{1}\right), \ldots, f\left(x_{t}, y_{t}\right)\right)$.

We adapt Lindell's protocol [14] to support our cut-and-choose technique in the parallel execution setting. The main difficulty is the design and construction of a generalization of cut-and-choose oblivious transfer [16] which we use to avoid the "selective failure attack" where a malicious $P_{1}$ constructs invalid keys for $P_{2}$ 's input wires to try and deduce $P_{2}$ 's inputs based on if $P_{2}$ aborts execution or not. We discuss this more in Section 2.1. We note that the naïve idea of using the XOR-tree approach [15] in our setting does not appear to work without using adaptively secure garbled circuits. Specifically, it is no longer clear how $P_{1}$, without any knowledge of which circuits will end up as evaluation circuits, can batch $P_{2}$ 's input keys together in a way that lets $P_{2}$ learn different sets of input keys corresponding to different evaluation circuits and yet within each evaluation bucket guaranteeing that $P_{2}$ can learn only input keys corresponding to the same set of inputs.

We give details of our protocol construction for the parallel executions setting in Section 2.2

\subsection{Generalizing Cut-and-Choose Oblivious Transfer}

Cut-and-choose oblivious transfer (C\&C OT) [16] is an extension of standard one-out-of-two oblivious transfer (OT). The sender inputs $L$ pairs of strings, and the receiver inputs $L$ selection bits to select one string out of each pair of sender strings. The receiver also inputs a set $J$ of size $L / 2$ that consists of indices 


\section{Inputs:}

- $P_{1}$ inputs $\ell$ vectors $\boldsymbol{x}_{i}$, each containing $s$ pairs of values $x_{0}^{i, j}, x_{1}^{i, j} \in\{0,1\}^{n \times n}$, $i \in[\ell], j \in[s]$. In addition, $P_{1}$ inputs $s$ "check values" $\chi_{1}, \ldots, \chi_{s} \in\left(\{0,1\}^{n}\right)^{s}$.

- $P_{2}$ inputs $\sigma_{1}, \ldots, \sigma_{\ell} \in\{0,1\}$ and a set of indices $J \subseteq[s]$.

Outputs: $P_{1}$ receives no output. $P_{2}$ receives the following:

- For every $i \in[\ell]$ and $j \in J, P_{2}$ receives $\left(x_{0}^{i, j}, x_{1}^{i, j}\right)$.

- For every $i \in[\ell], P_{2}$ receives $\left\langle x_{\sigma_{i}}^{i, 1}, \ldots, x_{\sigma_{i}}^{i, s}\right\rangle$.

- For every $k \notin J, P_{2}$ receives $\chi_{k}$.

In other words, $P_{2}$ receives $\left\{\chi_{j}\right\}_{j \in[s] \backslash J}$ and $\left\{\left\{x_{\sigma_{i}}^{i, j}\right\}_{j \in[s] \backslash J},\left\{\left(x_{0}^{i, j}, x_{1}^{i, j}\right)\right\}_{j \in J}\right\}_{i \in[\ell]}$.

Fig. 1. Modified batch single-choice cut-and-choose OT functionality $\mathcal{F}_{\text {ccot }} 14$

where it wants both the sender's inputs to be revealed. Note that for indices not contained in $J$, only those sender inputs that correspond to the receiver's selection bits are revealed. In applications to secure computation, and in particular when transferring input keys corresponding to a particular input wire across all evaluation circuits, one needs single-choice cut-and-choose oblivious transfer, where the receiver is restricted to inputting the same selection bit in all the $L / 2$ instances where it receives exactly one out of two sender strings. Furthermore, when transferring input keys for multiple input wires, it is crucial that the subset $J$ input by the receiver is the same across each instance of single-choice C\&C OT executed for all input wires. This variant, called batch single-choice $\mathrm{C} \& \mathrm{C}$ OT, can be realized from the decisional Diffie-Hellman problem [16].

Lindell [14] presented a variant of batch single-choice C\&C OT [16] in order to address settings where the check set $J$ input by the receiver may be of arbitrary size. We denote this variant by $\mathcal{F}_{\text {ccot }}$; see Figure 1 for the formal description. In this variant, in addition to obtaining one of the two sender inputs for pairs whose indices are not in $J$, the receiver also obtains a "check value" for each index not in $J$. These check values are used to confirm whether or not a circuit is an evaluation circuit.

For our purposes, we introduce a new variant of $\mathcal{F}_{\text {ccot }}$, which we call batch single-choice multi-stage C\&C OT. We denote this primitive by $\mathcal{F}_{\text {mcot }}$ and present its formal description in Figure 2. At a high level, our variant differs from $\mathcal{F}_{\text {ccot }}$ in that receiver $P_{2}$ can now input multiple sets $J_{1}, \ldots, J_{t}$ (where $J$ is now implicitly defined as $\left.[\rho t] \backslash \cup_{k \in[t]} J_{k}\right)$ and make independent selections for each of $J_{1}, \ldots, J_{t}$. Unlike in Lindell's scheme [14, we only need to consider sets $J_{1}, \ldots, J_{t}$ whose sizes are pre-specified in order to provide the desired security guarantees. However, as in the $\mathcal{F}_{\text {ccot }}$ functionality, $\mathcal{F}_{\text {mcot }}(1)$ does not require sets $J_{1}, \ldots, J_{t}$ to be of a particular size, and (2) delivers "check values" for indices contained in each of $J_{1}, \ldots, J_{t}$. These check values are used to confirm whether a circuit is an evaluation circuit in the $k$ th bucket for some $k \in[t]$. 


\section{Inputs:}

- $P_{1}$ inputs $\ell$ vectors $\boldsymbol{x}_{i}$, each containing $\rho t^{2}$ pairs $x_{0}^{i, j}, x_{1}^{i, j} \in\{0,1\}^{n}$. In addition, $P_{1}$ inputs $\rho t^{2}$ "check values" $\chi_{1}^{1}, \ldots, \chi_{\rho t}^{1} ; \ldots ; \chi_{1}^{t}, \ldots, \chi_{\rho t}^{t} \in\{0,1\}^{n}$.

- $P_{2}$ inputs $\boldsymbol{\sigma}_{1}=\left(\sigma_{1,1}, \ldots, \sigma_{1, \ell}\right), \ldots, \boldsymbol{\sigma}_{t}=\left(\sigma_{t, 1}, \ldots, \sigma_{t, \ell}\right) \in\{0,1\}^{\ell}$ and sets $J_{1}, \ldots, J_{t}$ that are pairwise non-intersecting subsets of $[\rho t]$.

Outputs: Party $P_{1}$ receives no output. Party $P_{2}$ receives the following:

- For every $k \in[t]$ and for every $j \in J_{k}$, party $P_{2}$ receives $\chi_{j}^{k}$.

- Let $J=[\rho t] \backslash \cup_{k \in[t]} J_{k}$. For every $i \in[\ell]$ and $j \in[\rho t]$ :

- If $j \in J$, then $P_{2}$ receives $\left(x_{0}^{i, j}, x_{1}^{i, j}\right)$.

- Otherwise, if there exists a (unique) $k \in[t]$ such that $j \in J_{k}$, then $P_{2}$ receives $x_{\sigma_{k, i}}^{i, j}$.

In other words, $P_{2}$ receives sets $\left\{\chi_{j}^{1}\right\}_{j \in J_{1}}, \ldots,\left\{\chi_{j}^{t}\right\}_{j \in J_{t}}$ and $\left\{\left\{x_{\sigma_{1, i}}^{i, j}\right\}_{j \in J_{1}}, \ldots\right.$, $\left.\left\{x_{\sigma_{t, i}}^{i, j}\right\}_{j \in J_{t}},\left\{\left(x_{0}^{i, j}, x_{1}^{i, j}\right)\right\}_{j \in J}\right\}_{i \in[\ell]}$.

Fig. 2. Batch single-choice multi-stage cut-and-choose OT functionality $\mathcal{F}_{\text {mcot }}$

Designing the $\mathcal{F}_{\text {mcot }}$ Functionality. As in $\mathcal{F}_{\text {ccot }}$, the sender $P_{1}$ inputs $\ell$ vectors $\boldsymbol{x}_{1}, \ldots, \boldsymbol{x}_{\ell}$ each of length $\rho t$, where each element in the vector is a pair of values (corresponding to the 0 -key and the 1-key of a given garbled wire). In addition, $P_{1}$ inputs $\rho t^{2}$ "check values". Receiver $P_{2}$ inputs $t$ vectors $\boldsymbol{\sigma}_{1}, \ldots, \boldsymbol{\sigma}_{t}$ each of length $\ell$ and pairwise non-intersecting sets $J_{1}, \ldots, J_{t}$. Upon receiving these inputs from $P_{1}$ and $P_{2}$, the functionality computes $J=[\rho t] \backslash \cup_{k \in[t]} I_{k}$, and delivers, for each $j \in J$, the $j$ th element (i.e., both values in the $j$ th pair) in each of the $\ell$ vectors. Next, for every $k \in[t]$ and for each $j \in J_{k}$, the functionality delivers to $P_{2}$ the $\sigma_{k, i}$ value in the $j$ th pair of vector $\boldsymbol{x}_{i}$ for every $i \in[\ell]$ along with the check value $\chi_{j}^{k}$.

Realizing $\mathcal{F}_{\text {mcot }}$ in the $\mathcal{F}_{\text {ccot-hybrid model. We now proceed to construct a pro- }}$ tocol for $\mathcal{F}_{\text {mcot }}$. Our goal is to provide an information-theoretic reduction from $\mathcal{F}_{\text {mcot }}$ to $\mathcal{F}_{\text {ccot }}$. We first consider a naïve approach which serves as a warm-up to our final construction and provides intuition behind our definition of $\mathcal{F}_{\text {mcot }}$.

The naïve approach. We propose the following natural approach to realizing $\mathcal{F}_{\text {mcot }}$ from $\mathcal{F}_{\text {ccot }}: P_{1}$ first performs a $t$-out-of- $t$ additive secret sharing of all input keys corresponding to $P_{2}$ 's inputs. In addition, $P_{1}$ chooses $\rho t^{2}$ check values. Next, $P_{1}$ and $P_{2}$ interact with the $\mathcal{F}_{\text {ccot }}$ functionality $t$ times in parallel. In the $k$ th interaction, $P_{1}$ provides the $k$ th additive share of its input plus $\rho t$ check values $\chi_{1}^{k}, \ldots, \chi_{\rho t}^{k}$ (i.e., a check value for each circuit that could potentially be an evaluation circuit in the $k$ th execution), while $P_{2}$ provides its inputs for the $k$ th execution along with a set $[\rho t] \backslash J_{k}$, where $J_{k}$ indicates the indices of the evaluation circuits to be used in the $k$ th execution. Let $J=[\rho t] \backslash \cup_{k \in[t]} J_{k}$. At the end of the interaction, $P_{2}$ obtains (1) all $t$ additive shares of input keys, and therefore all input keys, for circuits $G C_{j}$ with $j \in J$, and (2) all $t$ additive 
shares of input keys that correspond to its actual input in the $k$ th execution, and therefore its input keys, along with check values for circuits $G C_{j}$ with $j \notin J$.

Note, in particular, that for the check circuits, $P_{2}$ does not obtain the check values, and for the evaluation circuits, $P_{2}$ does not obtain both input keys. Thus, the above protocol seems to successfully fulfill our requirements from the $\mathcal{F}_{\text {mcot }}$ functionality. However, note that there is no mechanism in place to enforce that $P_{2}$ supplies non-intersecting sets $J_{1}, \ldots, J_{k}$. In the following we show that this prevents the above protocol from realizing $\mathcal{F}_{\text {mcot }}$.

Suppose $t=2$. A malicious $P_{2}$ may input overlapping sets $J_{1}, J_{2}$ to $\mathcal{F}_{\text {ccot }}$. The consequence of this is that $P_{2}$ now possesses check values $\chi_{j}^{1}$ and $\chi_{j}^{2}$ for $j \in J_{1} \cap J_{2}$. Clearly, the functionality $\mathcal{F}_{\text {mcot }}$ does not allow this. On the other hand, recall that the input keys are all additively shared, and as a result $P_{2}$ does not possess input keys corresponding to its input in circuit $G C_{j}$ unless its input in both executions are identical. At the surface, there does not seem to be any attack due to this malicious strategy. Sure, $P_{2}$ can now equivocate on assigning $G C_{j}$ to either the first evaluation bucket or the second evaluation. However, as observed earlier, it either has no corresponding keys, or it is going to evaluate both circuits on the same input, say $y$ (in which case it seems immaterial whether $j$ is revealed as part of $J_{1}$ or $J_{2}$ ). Unfortunately, we show that the above strategy for malicious $P_{2}$ is not simulatable. In particular, at the end of the interaction with $\mathcal{F}_{\text {ccot }}$, the simulator successfully extracts $P_{2}$ 's input in the first and second execution, but is now unable to decide on how to fake the garbled circuit $G C_{j}$. On the one hand, if $j \in J_{1}$, then the fake garbled circuit has to output $z_{1}=f\left(x_{1}, y\right)$. On the other hand, if $j \in J_{2}$, then the fake garbled circuit has to output $z_{2}=f\left(x_{2}, y\right)$. Therefore, the simulator has to choose on how to fake $G C_{j}$ in the dark. Note that a simulation strategy for this specific case that decides to fake $G C_{j}$ to output $z_{1}$ with probability $1 / 2$, and to output $z_{2}$ with probability $1 / 2$, does indeed succeed with probability $1 / 2$. However, this strategy does not extend well to the case when $t$ is large.

The discussion above motivates our definition of $\mathcal{F}_{\text {mcot }}$; in particular, it reinforces why $\mathcal{F}_{\text {mcot }}$ must deliver at most one check value per circuit. In the following, we explain how to modify the naïve construction to enforce this.

Our approach. The high level idea behind our protocol is to let $P_{1}$ perform independent additive sharings of both the input values as well as the check values. Then $P_{1}$ and $P_{2}$ query the $\mathcal{F}_{\text {ccot }}$ functionality $t$ times to transfer the values as required by $\mathcal{F}_{\text {mcot }}$. We detail this below, explaining it in the context of our secure computation protocol.

Let $\left(x_{0}^{i, j}, x_{1}^{i, j}\right)$ be the input keys corresponding to $P_{2}$ 's $i$ th input wire in $G C_{j}$. First, $P_{1}$ performs a $t$-out-of- $t$ additive secret sharing of all input values corresponding to $P_{2}$ 's inputs; i.e., for each $i \in[\ell], j \in[\rho t], P_{1}$ secret shares $x_{0}^{i, j}$ (resp., $x_{1}^{i, j}$ ) into $\left\{x_{0}^{i, j, k}\right\}_{k \in[t]}$ (resp., $\left\{x_{1}^{i, j, k}\right\}_{k \in[t]}$ ). $P_{1}$ then chooses $\rho t^{2}$ check values $\left\{\chi_{1}^{k}, \ldots, \chi_{\rho t}^{k}\right\}_{k \in[t]}$. It then performs a $(2 \ell(t-1)+1)$-out-of$(2 \ell(t-1)+1)$ additive sharing of each value $\chi_{j}^{k}$ to obtain shares denoted $\tilde{\chi}_{j}^{k}$,

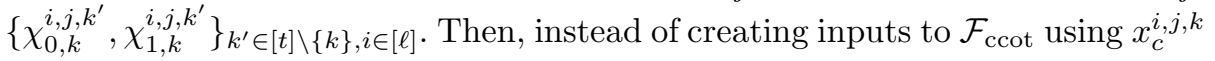


shares alone, $P_{1}$ instead creates a "share block" $X_{c}^{i, j, k}=\left(x_{c}^{i, j, k}, \chi_{c, 1}^{i, j, k}, \ldots, \chi_{c, t}^{i, j, k}\right)$. That is, a share block $X_{c}^{i, j, k}$ contains, in addition to a share of the input key, a share of all check values corresponding to circuit $G C_{j}$.

Next, $P_{1}$ and $P_{2}$ run $t$ instances of $\mathcal{F}_{\text {ccot }}$ in parallel. In the $k$ th interaction, in addition to the $\rho t$ check value shares $\widetilde{\chi}_{1}^{k}, \ldots, \widetilde{\chi}_{\rho t}^{k}, P_{1}$ provides its $k$ th share block while $P_{2}$ provides its inputs for the $k$ th execution along with a set $[\rho t] \backslash J_{k}$, where $J_{k}$ indicates the indices of the evaluation circuits to be used in the $k$ th execution. Let $J=[\rho t] \backslash \cup_{k \in[t]} J_{k}$. At the end of the interaction, $P_{2}$ obtains (1) all $t$ share blocks of input keys, and therefore all input keys, for circuits $G C_{j}$ with $j \in J$, and (2) all $t$ share blocks of input keys that correspond to its actual input in the $k$ th execution, and therefore its input keys, along with a check value $\tilde{\chi}_{j}^{k}$ for circuits $G C_{j}$ with $j \in J_{k}$.

Note, in particular, that for each check circuit $G C_{j}, P_{2}$ does not obtain the check value $\chi_{j}^{k}$ for any $k$, because it always misses the check value share $\tilde{\chi}_{j}^{k}$. For each evaluation circuit $G C_{j}$ with $j \in J_{k}, P_{2}$ does not obtain both input keys, and more importantly can obtain at most one check value (which is $\chi_{j}^{k}$ ). This is because share blocks contain shares of input keys as well as shares of check values. For an evaluation circuit, party $P_{2}$ always misses a share block, and consequently shares of all values $\chi_{j}^{k^{\prime}}$ with $k^{\prime} \neq k$. Furthermore, if $P_{2}$ wants to ensure it receives $\chi_{j}^{k}$, then it should never input $J_{k^{\prime \prime}}$ such that $k^{\prime \prime} \neq k$ and yet $j \in J_{k^{\prime \prime}}$. This is because for $j \in J_{k^{\prime \prime}}, P_{2}$ is guaranteed to miss a share block that contains an additive share of $\chi_{j}^{k}$. Note that the above observations suffice to deal with a malicious $P_{2}$ that inputs overlapping sets since in this case $P_{2}$ fails to obtain any check values corresponding to indices in the intersection.

The formal description of the protocol in the $\mathcal{F}_{\text {ccot }}$-hybrid model can be found in Figure 3. We prove the following in the full version.

Theorem 1. There exists a protocol perfectly realizing $\mathcal{F}_{\mathrm{mcot}}$ in the $\mathcal{F}_{\mathrm{ccot}}$-hybrid model.

\subsection{Using $\mathcal{F}_{\text {mcot }}$ in the Parallel Execution Setting}

The input vectors $\boldsymbol{x}_{i}$, for $i \in[\ell]$, contain the key pairs associated with the $i$ th input wire for $P_{2}$ in each of the $\rho t$ circuits. The vector $\boldsymbol{\sigma}_{k}$ corresponds to the inputs used by $P_{2}$ in the $k$ th execution. An honest $P_{2}$ chooses sets $J_{1}, \ldots, J_{t}$ such that they are pairwise non-intersecting and each set is of size exactly $\rho / 2$. The main observation is that, for a given execution $k \in[t], P_{2}$ obtains check values $\chi_{j}^{k}$ from $\mathcal{F}_{\text {mcot }}$ only for $j \in J_{k}$. Therefore, once the parties complete the interaction with $\mathcal{F}_{\text {mcot }}$ and $P_{1}$ sends all the garbled circuits, we let $P_{1}$ determine the evaluation circuits in each bucket based on whether $P_{2}$ sends the corresponding check values. At this point, $P_{1}$ checks that each bucket of evaluation circuits is well-defined and that these buckets are of equal size, i.e., $\rho / 2$. If not, $P_{1}$ aborts. To overcome technical difficulties, we also require $P_{2}$ to provide "check values" for the check circuits as well. A check value for check circuit $G C_{j}$, denoted $\chi_{j}$, may simply be the set of all input keys (i.e., both the 0-key and the 1-key) on all wires in circuit $G C_{j}$. 


\section{Inputs:}

- $P_{1}$ inputs $\ell$ vectors of pairs $\boldsymbol{x}_{i}=\left\langle\left(x_{0}^{i, 1}, x_{1}^{i, 1}\right), \ldots,\left(x_{0}^{i, \rho t}, x_{1}^{i, \rho t}\right)\right\rangle$ for $i \in[\ell]$. In addition, $P_{1}$ inputs $\rho t^{2}$ "check values" $\left(\chi_{1}^{1}, \ldots, \chi_{\rho t}^{1}\right), \ldots,\left(\chi_{1}^{t}, \ldots, \chi_{\rho t}^{t}\right)$. All values are in $\{0,1\}^{n}$.

- $P_{2}$ inputs $\boldsymbol{\sigma}_{1}=\left(\sigma_{1,1}, \ldots, \sigma_{1, \ell}\right), \ldots, \boldsymbol{\sigma}_{t}=\left(\sigma_{t, 1}, \ldots, \sigma_{t, \ell}\right) \in\{0,1\}^{\ell}$ and sets $J_{1}, \ldots, J_{t}$.

\section{Protocol:}

- For all $i \in[\ell], P_{1}$ performs a $t$-out-of- $t$ additive secret sharing of $\boldsymbol{x}_{i}$ to obtain shares $\boldsymbol{x}_{i, 1}, \ldots, \boldsymbol{x}_{i, t}$. For $k \in[t]$, let $\boldsymbol{x}_{i, k}=\left\langle\left(x_{0}^{i, 1, k}, x_{1}^{i, 1, k}\right), \ldots,\left(x_{0}^{i, \rho t, k}, x_{1}^{i, \rho t, k}\right)\right\rangle$. Let $X_{0}^{i, j, k}=\left(x_{0}^{i, j, k}, \chi_{0,1}^{i, j, k}, \ldots, \chi_{0, t}^{i, j, k}\right)$ and $X_{1}^{i, j, k}=\left(x_{1}^{i, j, k}, \chi_{1,1}^{i, j, k}, \ldots, \chi_{1, t}^{i, j, k}\right)$, where $\chi_{0,1}^{i, j, k}, \ldots, \chi_{0, t}^{i, j, k}$ and $\chi_{1,1}^{i, j, k}, \ldots, \chi_{1, t}^{i, j, k}$ are random independent values in $\{0,1\}^{n}$. Let $\boldsymbol{X}_{i, k}=\left\langle\left(X_{0}^{i, 1, k}, X_{1}^{i, 1, k}\right), \ldots,\left(X_{0}^{i, \rho t, k}, X_{1}^{i, \rho t, k}\right)\right\rangle$.

- For all $k \in[t]$ and $j \in[\rho t]$, set $\tilde{\chi}_{j}^{k}=\chi_{j}^{k} \oplus \bigoplus_{k^{\prime} \in[t] \backslash\{k\}, i \in[\ell]}\left(\chi_{0, k}^{i, j, k^{\prime}} \oplus \chi_{1, k}^{i, j, k^{\prime}}\right)$.

- $P_{1}$ and $P_{2}$ run $t$ instances of $\mathcal{F}_{\text {ccot }}$ in parallel as follows. In the $k$ th instance:

- $P_{1}$ inputs $\ell$ vectors of pairs $\boldsymbol{X}_{i, k}$ of length $\rho t$ for $i \in[\ell]$ and $\rho t$ "check values" $\tilde{\chi}_{1}^{k}, \ldots, \tilde{\chi}_{\rho t}^{k}$. $P_{2}$ inputs $\sigma_{k, 1}, \ldots, \sigma_{k, \ell} \in\{0,1\}$ and the set $[\rho t] \backslash J_{k}$.

- $P_{2}$ receives $\left\{\tilde{\chi}_{j}^{k}\right\}_{j \in J_{k}}$ and $\left\{\left\{X_{\sigma_{k, i}}^{i, j, k}\right\}_{j \in J_{k}} \cup\left\{\left(X_{0}^{i, j, k}, X_{1}^{i, j, k}\right)\right\}_{j \in[\rho t] \backslash J_{k}}\right\}_{i \in[\ell]}$.

- For all $k \in[t]$ and $j \in J_{k}, P_{2}$ reconstructs $\chi_{j}^{k}=\tilde{\chi}_{j}^{k} \oplus \bigoplus_{k^{\prime} \in[t] \backslash\{k\}, i \in[\ell]}\left(\chi_{0, k}^{i, j, k^{\prime}}\right.$ $\left.\oplus \chi_{1, k}^{i, j, k^{\prime}}\right)$.

- Let $J=[\rho t] \backslash \cup_{k \in[t]} J_{k}$. For all $i \in[\ell]$ and $j \in[\rho t], P_{2}$ does the following:

- If $j \in J$ : set $x_{0}^{i, j}=\bigoplus_{k \in[t]} x_{0}^{i, j, k}$, and $x_{1}^{i, j}=\bigoplus_{k \in[t]} x_{1}^{i, j, k}$.

- If there exists (unique) $k \in[t]$ such that $j \in J_{k}$ : set $x_{\sigma_{k, i}}^{i, j}=\bigoplus_{k \in[t]} x_{\sigma_{k, i}}^{i, j, k}$.

- $P_{2}$ outputs sets $\left\{\chi_{j}^{1}\right\}_{j \in J_{1}}, \ldots,\left\{\chi_{j}^{t}\right\}_{j \in J_{t}}$ and $\left\{\left\{\left(x_{0}^{i, j}, x_{1}^{i, j}\right)\right\}_{j \in J},\left\{x_{\sigma_{1, i}}^{i, j}\right\}_{j \in J_{1}}, \ldots\right.$, $\left.\left\{x_{\sigma_{t, i}}^{i, j}\right\}_{j \in J_{t}}\right\}_{i \in[\ell]}$.

Fig. 3. Realizing $\mathcal{F}_{\text {mcot }}$ in the $\mathcal{F}_{\text {ccot-hybrid model }}$

Applying the Cheating-Punishment Technique. Inspired by Lindell's protocol [14, we use the knowledge of two different garbled values for a single output wire as a "proof" that $P_{2}$ received inconsistent outputs in a given execution. $P_{2}$ can use this proof to obtain $P_{1}$ 's input in a cheating-punishment phase. This cheating-punishment phase is implemented via a secure computation protocol, and thus it is important that the second phase functionality has a small circuit. We employ several optimizations proposed by Lindell [14] to keep the size of this circuit small. One important difference in our setting is that, unlike in Lindell's protocol 14, we cannot have, for a given output wire $w$, the same output keys $b_{w}^{0}, b_{w}^{1}$ across all garbled circuits. This is because in our setting garbled circuits are assigned to different evaluation buckets, and the circuits in each bucket can be evaluated with different input values, and thus can produce different outputs. Thus (even an honest) $P_{2}$ could potentially learn, say, output key $b_{w}^{0}$ in one execution and output key $b_{w}^{1}$ in another. We address this by simply removing the 
requirement that the set of output keys across different garbled circuits are the same. Thus, the circuit for the cheating-punishment phase for the $k$ th execution must now take as input from $P_{1}$ all of the output keys in all of the evaluation circuits in the $k$ th bucket, and from $P_{2}$ a pair of output keys that serve as proof of cheating. Somewhat surprisingly, we show that the size of the circuit (measured as the number of non-XOR gates) for the cheating-punishment phase is essentially the same as the circuit in Lindell's protocol [14] 2

Another detail we wish to point out is that in our protocol we need to run separate cheating-punishment phases for each execution. This is a restriction imposed by the way in which $P_{1}$ proves consistency of its inputs [14 16]. However, we can run all of the $t$ cheating-punishment phases in parallel. For this reason we use the universally composable variant of Lindell and Pinkas's protocol [16] (which is essentially obtained by replacing oblivious transfers and zero-knowledge subprotocols with their universally composable variants) to implement each cheating-punishment phase.

Other Details. We now describe other important details of our protocol.

- Input consistency across multiple executions. It is important to guarantee that $P_{1}$ provides consistent inputs across all circuits in the $k$ th execution. Fortunately, existing mechanisms [1416] for ensuring input consistency in the single execution setting can be readily extended to the multiple execution setting as well.

- Encoded translation tables for garbled circuits. As in Lindell's protocol [14, we modify the output translation tables used in the garbled circuits. Specifically, for keys $k_{i}^{0}, k_{i}^{1}$ on output wire $i$, we create an encoded output table $\left[h\left(k_{i}^{0}\right), h\left(k_{i}^{1}\right)\right]$, where $h$ is some one-way function. We require that the output keys (or more precisely, the output of $h$ applied to the output keys) corresponding to 0 and 1 are distinct. This encoding gives us the following two properties: (1) $P_{2}$ after evaluating a garbled circuit can use the encoded translation tables to determine whether the output is 0 or 1 , and (2) the encoded translation table does not reveal the other output key (since this is equivalent to inverting the one-way function) to $P_{2}$.

- Optimizing the cheating-punishment circuit. We can apply similar techniques as shown by Lindell [14] to optimize the size of the cheating-punishment circuit to contain only $\ell$ non-XOR gates. We leave the details to the full version.

Formal Description. We proceed to the formal description of our protocol.

Inputs: $P_{1}$ has input $x=\left(x_{1}, \ldots, x_{t}\right)$, where $x_{k} \in\{0,1\}^{\ell}$, and $P_{2}$ has input $y=\left(y_{1}, \ldots, y_{t}\right)$, where $y_{k} \in\{0,1\}^{\ell}$.

${ }^{2}$ Of course, the cost of realizing our cheating-punishment phase is more than the corresponding cost in Lindell's protocol [14], mainly due to $P_{1}$ 's input being larger (but only by a factor of $\rho / 2$ ). 
Auxiliary Inputs: A statistical security parameter $s$, a computational security parameter $n$, the description of a circuit $C$ where $C(x, y)=f(x, y)$, the number of evaluations $t$ of the function $f$, and $(\mathbb{G}, q, g)$ where $\mathbb{G}$ is a cyclic group with generator $g$ and prime order $q$, where $q$ is of length $n$. Let Ext : $\mathbb{G} \rightarrow\{0,1\}^{n}$ be a function mapping group elements to bitstrings. In the following, $\rho=\rho(s, t)$ is the replication factor defined as being the smallest $u \in \mathbb{N}$ such that for all $m \in\{u / 2, \ldots, u t / 2\}$ it holds that $t \cdot\left(\begin{array}{c}u t-m \\ u t / 2\end{array}\right)\left(\begin{array}{c}m \\ u / 2\end{array}\right) /\left(\begin{array}{c}u t \\ u t / 2\end{array}\right)\left(\begin{array}{c}u t / 2 \\ u / 2\end{array}\right) \leq 2^{-s}$. If no such $u$ exists or if $\rho \geq s$, then parties abort this protocol, and instead run the fast C\&C protocol [14] for the function $f^{(t)}$.

Outputs: $P_{2}$ receives $f^{(t)}(x, y)$ and $P_{1}$ receives no output. Let $\ell^{\prime}$ denote the length of the output of $f(x, y)$.

\section{Protocol:}

1. Input key choice and circuit preparation:

- $P_{1}$ chooses random values $a_{1}^{0}, a_{1}^{1}, \ldots, a_{\ell}^{0}, a_{\ell}^{1} \in \in_{R} \mathbb{Z}_{q}, r_{1}, \ldots, r_{\rho t} \in_{R} \mathbb{Z}_{q}$ and $\left(b_{1,1}^{0}, b_{1,1}^{1}, \ldots, b_{1, \ell^{\prime}}^{0}, b_{1, \ell^{\prime}}^{1}\right), \ldots,\left(b_{\rho t, 1}^{0}, b_{\rho t, 1}^{1}, \ldots, b_{\rho t, \ell^{\prime}}^{0}, b_{\rho t, \ell^{\prime}}^{1}\right) \in_{R}\{0,1\}^{n \ell^{\prime}}$ such that for every $c_{1}, c_{2} \in\{0,1\}, j_{1}, j_{2} \in[\rho t], i_{1}, i_{2} \in\left[\ell^{\prime}\right]$ it holds that $b_{j_{1}, i_{1}}^{c_{1}}=b_{j_{2}, i_{2}}^{c_{2}}$ iff $i_{1}=i_{2}$ and $j_{1}=j_{2}$ and $c_{1}=c_{2}$.

- Let $w_{1}, \ldots, w_{\ell}$ denote the input wires corresponding to $P_{1}$ 's input, let $w_{i, j}$ denote the $i$ th input wire in the $j$ th garbled circuit, and let $k_{i, j}^{b}$ denote the key associated with bit $b$ on wire $w_{i, j} . P_{1}$ sets $k_{i, j}^{b}$ as follows:

$$
k_{i, j}^{0}=\operatorname{Ext}\left(g^{a_{i}^{0} \cdot r_{j}}\right) \quad \text { and } \quad k_{i, j}^{1}=\operatorname{Ext}\left(g^{a_{i} \cdot r_{j}}\right) \text {. }
$$

- Let $w_{1}^{\prime}, \ldots, w_{\ell^{\prime}}^{\prime}$ denote the output wires. The keys for wire $w_{i}^{\prime}$ in the $j$ th garbled circuit are set to $b_{j, i}^{0}$ and $b_{j, i}^{1}$.

- $P_{1}$ constructs $\rho t$ independent garblings, $G C_{1}, \ldots, G C_{\rho t}$, of circuit $C$, using random keys except for wires $w_{1}, \ldots, w_{\ell}$ and $w_{1}^{\prime}, \ldots, w_{m}^{\prime}$, where the keys are set as above.

2. Oblivious transfers: $P_{1}$ and $P_{2}$ run $\mathcal{F}_{\text {mcot }}$ as follows:

- For $i \in[\ell]$, let $\boldsymbol{z}_{i}$ denote a vector containing the $\rho t$ pairs of keys associated with $P_{2}$ 's $i$ th input bit in all the garbled circuits. $P_{1}$ inputs $\boldsymbol{z}_{1}, \ldots, \boldsymbol{z}_{\ell}$, as well as random values $\chi_{1}^{1}, \ldots, \chi_{\rho t}^{1} ; \ldots ; \chi_{1}^{t}, \ldots, \chi_{\rho t}^{t}$.

- $P_{2}$ inputs random sets $J_{1}, \ldots, J_{t}$ which are pairwise non-intersecting subsets of $[\rho t]$ such that for all $k \in[t]$ it holds that $\left|J_{k}\right|=\rho / 2$. Let $J=[\rho t] \backslash \cup_{k \in[t]} J_{k} . P_{2}$ also inputs bits $\left(\sigma_{1,1}, \ldots, \sigma_{1, \ell}\right), \ldots,\left(\sigma_{t, 1}, \ldots, \sigma_{t, \ell}\right) \in$ $\{0,1\}^{\ell}$, where $\sigma_{k, i}=y_{k, i}$ for every $i \in[\ell]$ and $k \in[t]$.

- For $j \in J, P_{2}$ receives both input keys associated with its input wires in garbled circuit $G C_{j}$, and for each $k \in[t]$ and $j \in J_{k}, P_{2}$ receives the keys associated with its input $y_{k}$ on its input wires in garbled circuit $G C_{j}$. Also, for every $k \in[t]$ and $j \in J_{k}, P_{2}$ receives $\chi_{j}^{k}$.

3. Send circuits and commitments: $P_{1}$ sends $P_{2}$ the garbled circuits $G C_{1}, \ldots, G C_{\rho t}$, the "seed" for the randomness extractor Ext, the following commitment to the garbled values associated with $P_{1}$ 's input wires:

$$
\left\{\left(i, 0, g^{a_{i}^{0}}\right),\left(i, 1, g^{a_{i}^{1}}\right)\right\}_{i \in[\ell]} \quad \text { and } \quad\left\{\left(j, g^{r_{j}}\right)\right\}_{j=1}^{\rho t}
$$

and the encoded output translation tables:

$$
\left\{\left[\left(h\left(b_{j, 1}^{0}\right), h\left(b_{j, 1}^{1}\right)\right), \ldots,\left(h\left(b_{j, \ell^{\prime}}^{0}\right), h\left(b_{j, \ell^{\prime}}^{1}\right)\right)\right]\right\}_{j \in[\rho t]} .
$$

If $h\left(b_{j, i}^{0}\right)=h\left(b_{j, i}^{1}\right)$ for any $1 \leq i \leq \ell^{\prime}, 1 \leq j \leq \rho t$, then $P_{2}$ aborts. 
4. Send cut-and-choose challenge: $P_{2}$ sends $P_{1}$ the sets $J, J_{1}, \ldots, J_{t}$ along with values $\left\{\chi_{j}^{1}\right\}_{j \in J_{1}}, \ldots,\left\{\chi_{j}^{t}\right\}_{j \in J_{t}}$, and all the keys associated with its input wires in all circuits $G C_{j}$ for $j \in J$. If the values received by $P_{1}$ are (1) incorrect, or (2) the sets $J_{1}, \ldots, J_{t}$ are not pairwise non-intersecting, or (3) the input keys associated with $P_{2}$ 's input wires in circuits $G C_{j}$ are revealed incorrectly, or (4) there exists some $k \in[t]$ such that $\left|J_{k}\right| \neq \rho / 2$, then it outputs $\perp$ and aborts. Circuits $G C_{j}$ for $j \in J$ are called check circuits and circuits $G C_{j}$ for $j \in J_{k}$ are called evaluation circuits in the kth bucket.

5. Send garbled input values in the evaluation circuits: For each $k \in[t]$ : $P_{1}$ sends the input keys associated with input $x_{k}$ for the evaluation circuits in the $k$ th bucket: For each $j \in J_{k}$ and every wire $i \in[\ell], P_{1}$ sends the value $k_{i, j}^{\prime}=g^{a_{i}{ }_{k, i}} \cdot r_{j}$ and $P_{2}$ sets $k_{i, j}=\operatorname{Ext}\left(k_{i, j}^{\prime}\right)$.

6. Circuit evaluation: For each $k \in[t], P_{2}$ does the following:

- For each $j \in J_{k}$ and every wire $i \in\left[\ell^{\prime}\right], P_{2}$ computes $b_{j, i}^{\prime}$ by evaluating $G C_{j}$. If $P_{2}$ receives exactly one valid output value per output wire, then let $z_{k}$ denote this output. In this case, it chooses random values $b_{0}^{k}, b_{1}^{k} \in_{R}$ $\{0,1\}^{n}$. If $P_{2}$ receives two valid outputs on any output wire then it sets $b_{0}^{k}=b_{j_{1}, i}^{\prime}$ and $b_{1}^{k}=b_{j_{2}, i}^{\prime}$, where $j_{1}, j_{2} \in J_{k}$ denote the conflicting circuit indices. If $P_{2}$ receives no valid output values on any output wire, then $P_{2}$ aborts.

7. Run secure computation to detect cheating: For each $k \in[t], P_{1}$ and $P_{2}$ do the following in parallel:

$P_{1}$ defines a circuit with the values $\left\{b_{j, 1}^{0}, b_{j, 1}^{1}, \ldots, b_{j, \ell^{\prime}}^{0}, b_{j, \ell^{\prime}}^{1}\right\}_{j \in J_{k}}$ hardcoded.

The circuit computes the following function:

- $P_{1}$ inputs $x_{k} \in\{0,1\}^{\ell}$ and has no output.

- $P_{2}$ inputs a pair of values $b_{0}^{k}, b_{1}^{k}$.

- If there exists values $i \in\left[\ell^{\prime}\right]$ and $j_{1}, j_{2} \in J_{k}$ such that $b_{0}^{k}=b_{j_{1}, i}^{0}$ and $b_{1}^{k}=b_{j_{2}, i}^{1}$, then $P_{2}$ 's output is $x_{k}$; otherwise it receives no output.

$P_{1}$ and $P_{2}$ run the UC-secure protocol of Lindell and Pinkas [16] on this circuit (except for the proof of $P_{1}$ 's input values), as follows:

- $P_{1}$ inputs $x_{k} ; P_{2}$ inputs $b_{0}^{k}$ and $b_{1}^{k}$ as computed in Step 6.

- The garbled circuits constructed by $P_{1}$ use the same $a_{i}^{0}, a_{i}^{1}$ values as were chosen in Step 1, and the parties use $3(s+\log t)$ copies of the circuit for the cut-and-choose.

If this computation results in an abort, then both parties halt.

8. Check circuits for computing $f^{(t)}(x, y)$ :

- For $j \in J, P_{1}$ sends $r_{j}$ to $P_{2}$, and $P_{2}$ checks that these values are consistent with the pairs $\left\{\left(j, g^{r_{j}}\right)\right\}_{j \in J}$ received in Step 3. If not, $P_{2}$ aborts.

- For every $j \in J, P_{2}$ uses the $g^{a_{i}^{0}}, g^{a_{i}^{1}}$ values received in Step 3 and the $r_{j}$ values received above to compute the keys for $P_{1}$ 's input wires as $k_{i, j}^{0}=$ $\operatorname{Ext}\left(g^{a_{i}^{0} \cdot r_{j}}\right), k_{i, j}^{1}=\operatorname{Ext}\left(g^{a_{i}^{1} \cdot r_{j}}\right)$. In addition, $P_{2}$ uses the keys obtained from $\mathcal{F}_{\text {mcot }}$ in Step 2 for its own input wires. $P_{2}$ verifies that $G C_{j}$ is a correct garbling of $C$. If there exists a circuit for which this does not hold, then $P_{2}$ aborts.

9. Verify consistency of $P_{1}$ 's input: For each $k \in[t]$ : Let $\widehat{J}_{k}$ be the set of check circuits used in the $2 \mathrm{PC}$ computation in Step 7 for the $k$ th bucket, let $\widehat{r}_{j, k}$ be the value used in that computation, and let $\hat{k}_{i, j}$ be the analogous value of $k_{i, j}^{\prime}$ in Step 5 received by $P_{2}$ in the computation in Step 7. For each $k \in[t]$, $P_{1}$ and $P_{2}$ do the following in parallel: 
- For every input wire $i \in\left[\ell^{\prime}\right], P_{1}$ proves a zero-knowledge proof-ofknowledge that there exist some $\sigma_{k, i} \in\{0,1\}$ such that for every $j \in J_{k}$ and every $j^{\prime} \notin \hat{J}_{k}$, it holds that $k_{i, j}^{\prime}=g^{a_{i}{ }^{{ }_{k}, i} \cdot r_{j}}$ and $\hat{k}_{i, j}=g^{a_{i}^{\sigma_{k, i}} \cdot \hat{r}_{j^{\prime}, k}}$. If any of the $t$ proofs fail, then $P_{2}$ aborts.

10. Output evaluation: For each $k \in[t], P_{2}$ does the following:

- If $P_{2}$ received no inconsistent outputs in Step 6, then it uses the encoded translation tables to decode the outputs it received, and sets $z_{k}$ to that value. If $P_{2}$ received inconsistent output, then let $x_{k}$ be the output that $P_{2}$ received from the circuit in Step 7 . Let $z_{k}=f\left(x_{k}, y_{k}\right)$ be the output in this case.

$P_{2}$ outputs $z=\left(z_{1}, \ldots, z_{t}\right)$ and terminates.

We prove the following theorem in the full version.

Theorem 2. Let $s$ (resp., $n$ ) be the statistical (resp., computational) security parameter. If the decisional Diffie-Hellman assumption holds in $(\mathbb{G}, g, q), h$ is a one-way function, and the underlying circuit garbling procedure is secure, then for all $t=\operatorname{poly}(n)$, the protocol described above securely computes $f^{(t)}$ in the presence of a malicious adversary with error at most $2^{-s}+\mu(n)$ for some negligible function $\mu(\cdot)$.

\section{The Sequential Execution Setting}

We now consider the setting where the parties securely evaluate the same function $f$ multiple times sequentially. Let $t$ denote the number of times the parties wish to evaluate $f$. Let $P_{1}$ 's (resp., $P_{2}$ 's) input in the $k$ th execution be denoted by $x_{k}$ (resp., $\left.y_{k}\right)$. Let $f^{[t]}$ denote the reactive functionality that computes $f$ a total of $t$ times sequentially.

The main difference between this setting and the parallel setting discussed in Section 2 is that in the sequential setting the parties may not know their inputs to all executions at the start of the protocol. In particular, inputs may depend on outputs from previous executions. Thus, the parallel execution protocol does not immediately carry over to the sequential setting. To see why, observe for instance that $\mathcal{F}_{\text {mcot }}$ requires $P_{2}$ to submit all of its inputs at onc 3 . This is not possible since in the sequential setting we cannot assume that $P_{2}$ has all its inputs at the beginning of the protocol. Instead, we take a different route; namely, we use the "XOR-tree" approach [15/26] to protect against the so-called "selective failure attack" 131925 . (In the parallel execution setting, this attack was implicitly avoided due to the use of $\mathcal{F}_{\text {mcot }}$.) In this approach, the circuit $C$ to be evaluated is first modified into an equivalent circuit $C_{\mathrm{XT}}$ (to include an "XOR-tree" for

\footnotetext{
${ }^{3}$ Standard oblivious transfer precomputation/"correction" techniques [2] still apply to $\mathcal{F}_{\text {mcot }}$ as well; however, it is not clear how to "correct" $\mathcal{F}_{\text {mcot }}$ correlations in a way suitable for the sequential setting.
} 
$P_{2}$ 's inputs). Then, $P_{1}$ sends commitments to input keys corresponding to $P_{2}$ 's input wires in $C_{\mathrm{XT}}$. The corresponding decommitments are revealed to $P_{2}$ via a standard one-out-of-two oblivious transfer. In order to prevent $P_{2}$ from using different inputs across evaluation circuits within the same bucket, $P_{1}$ batches together the decommitments corresponding to a particular input wire across all evaluation circuits in a given bucket. Note that herein lies an opportunity for a malicious $P_{1}$ to force $P_{2}$ to abort the protocol depending on its input. (This can be done for instance by sending incorrect decommitments for say only the 0-key on a particular wire.) However, the modified circuit $C_{\mathrm{XT}}$ is such that the success of any such selective OT attack is statistically independent of $P_{2}$ 's actual input value. Therefore, if an honest $P_{2}$ receives an invalid decommitment and is unable to decrypt the evaluation circuit, then it simply aborts knowing that its privacy is not compromised. Finally, we note that since we use one-out-oftwo oblivious transfer (as opposed to $\mathcal{F}_{\text {mcot }}$ ), we can leverage oblivious transfer extension techniques [101121] to obtain better efficiency.

We stress that the oblivious transfer step happens after $P_{1}$ sends all the GCs to $P_{2}$. This is because $P_{2}$ 's inputs to all $t$ executions are not available at the beginning of the protocol. Further, $P_{2}$ 's inputs may depend on previous outputs, which can be obtained only by decrypting evaluation circuits, i.e., after the evaluation bucket for the current execution is fully determined. Note that our cut-and-choose technique guarantees that there is at least one good evaluation circuit in every bucket under the assumption that $P_{1}$ has already committed to all its (good and bad) garbled circuits before the check sets and the evaluation sets are determined. Unfortunately, the above ordering of the oblivious transfer step and the garbled circuit sending step now allows a malicious $P_{2}$ to choose its input as a function of the garbled circuits it receives. To counter this, we need to use adaptively secure garbling schemes 3. instead of standard garbled circuits; adaptively secure garbling schemes can be constructed efficiently in the programmable random oracle model [3. Note that we do not need the use of adaptively secure garbling schemes for implementing the cheating-punishment phase. Indeed, all the inputs for that subprotocol are known before the phase begins, and therefore, the oblivious transfer step can be carried out before $P_{1}$ sends its garbled circuits for that phase.

Due to lack of space, we leave both the formal description and the proof of the following theorem to the full version.

Theorem 3. Let $s$ (resp., $n$ ) be the statistical (resp., computational) security parameter. If the decisional Diffie-Hellman assumption holds in $(\mathbb{G}, g, q), h$ is a one-way function, and the circuit is garbled using an adaptively secure garbling scheme, then for all polynomial values of $t$, the protocol described above securely computes $f^{[t]}$ in the presence of a malicious adversary with error at most $2^{-s}+$ $\mu(n)$ for some negligible function $\mu(\cdot)$.

Acknowledgments. Work of Yan Huang and Jonathan Katz supported in part by NSF award \#1111599. Work of Vladimir Kolesnikov supported in part by the Intelligence Advanced Research Project Activity (IARPA) via Department 
of Interior National Business Center (DoI/NBC) contract Number D11PC20194. Work of Ranjit Kumaresan supported by funding from the European Community's Seventh Framework Programme (FP7/2007-2013) under grant agreement number 259426. Work of Alex J. Malozemoff conducted with Government support through the National Defense Science and Engineering Graduate (NDSEG) Fellowship, 32 CFG 168a, awarded by DoD, Air Force Office of Scientific Research. The U.S. Government is authorized to reproduce and distribute reprints for Governmental purposes notwithstanding any copyright annotation thereon. Disclaimer: The views and conclusions contained herein are those of the authors and should not be interpreted as necessarily representing the official policies or endorsements, either expressed or implied, of IARPA, DoI/NBC, or the U.S. Government.

\section{References}

1. Applebaum, B., Ishai, Y., Kushilevitz, E., Waters, B.: Encoding functions with constant online rate or how to compress garbled circuits keys. In: Canetti, R., Garay, J.A. (eds.) CRYPTO 2013, Part II. LNCS, vol. 8043, pp. 166-184. Springer, Heidelberg (2013)

2. Beaver, D.: Precomputing oblivious transfer. In: Coppersmith, D. (ed.) CRYPTO 1995. LNCS, vol. 963, pp. 97-109. Springer, Heidelberg (1995)

3. Bellare, M., Hoang, V.T., Rogaway, P.: Adaptively secure garbling with applications to one-time programs and secure outsourcing. In: Wang, X., Sako, K. (eds.) ASIACRYPT 2012. LNCS, vol. 7658, pp. 134-153. Springer, Heidelberg (2012)

4. Cash, D., Jarecki, S., Jutla, C., Krawczyk, H., Roşu, M.-C., Steiner, M.: Highlyscalable searchable symmetric encryption with support for boolean queries. In: Canetti, R., Garay, J.A. (eds.) CRYPTO 2013, Part I. LNCS, vol. 8042, pp. 353373. Springer, Heidelberg (2013)

5. Damgård, I., Orlandi, C.: Multiparty computation for dishonest majority: From passive to active security at low cost. In: Rabin, T. (ed.) CRYPTO 2010. LNCS, vol. 6223, pp. 558-576. Springer, Heidelberg (2010)

6. Frederiksen, T.K., Jakobsen, T.P., Nielsen, J.B., Nordholt, P.S., Orlandi, C.: MiniLEGO: Efficient secure two-party computation from general assumptions. In: Johansson, T., Nguyen, P.Q. (eds.) EUROCRYPT 2013. LNCS, vol. 7881, pp. 537556. Springer, Heidelberg (2013)

7. Gennaro, R., Gentry, C., Parno, B.: Non-interactive verifiable computing: Outsourcing computation to untrusted workers. In: Rabin, T. (ed.) CRYPTO 2010. LNCS, vol. 6223, pp. 465-482. Springer, Heidelberg (2010)

8. Gordon, S.D., Katz, J., Kolesnikov, V., Krell, F., Malkin, T., Raykova, M., Vahlis, Y.: Secure two-party computation in sublinear (amortized) time. In: Yu, T., Danezis, G., Gligor, V.D. (eds.) ACM CCS 2012, pp. 513-524. ACM Press (2012)

9. Huang, Y., Katz, J., Evans, D.: Efficient secure two-party computation using symmetric cut-and-choose. In: Canetti, R., Garay, J.A. (eds.) CRYPTO 2013, Part II. LNCS, vol. 8043, pp. 18-35. Springer, Heidelberg (2013)

10. Ishai, Y., Kilian, J., Nissim, K., Petrank, E.: Extending oblivious transfers efficiently. In: Boneh, D. (ed.) CRYPTO 2003. LNCS, vol. 2729, pp. 145-161. Springer, Heidelberg (2003) 
11. Ishai, Y., Prabhakaran, M., Sahai, A.: Founding cryptography on oblivious transfer - efficiently. In: Wagner, D. (ed.) CRYPTO 2008. LNCS, vol. 5157, pp. 572-591. Springer, Heidelberg (2008)

12. Jarecki, S., Jutla, C.S., Krawczyk, H., Rosu, M.C., Steiner, M.: Outsourced symmetric private information retrieval. In: Sadeghi, A.R., Gligor, V.D., Yung, M. (eds.) ACM CCS 2013, pp. 875-888. ACM Press (2013)

13. Kiraz, M., Schoenmakers, B.: A protocol issue for the malicious case of Yao's garbled-circuit construction. In: 27th Symposium on Information Theory in the Benelux, pp. 283-290 (2006)

14. Lindell, Y.: Fast cut-and-choose based protocols for malicious and covert adversaries. In: Canetti, R., Garay, J.A. (eds.) CRYPTO 2013, Part II. LNCS, vol. 8043, pp. 1-17. Springer, Heidelberg (2013)

15. Lindell, Y., Pinkas, B.: An efficient protocol for secure two-party computation in the presence of malicious adversaries. In: Naor, M. (ed.) EUROCRYPT 2007. LNCS, vol. 4515, pp. 52-78. Springer, Heidelberg (2007)

16. Lindell, Y., Pinkas, B.: Secure two-party computation via cut-and-choose oblivious transfer. In: Ishai, Y. (ed.) TCC 2011. LNCS, vol. 6597, pp. 329-346. Springer, Heidelberg (2011)

17. Lindell, Y., Riva, B.: Cut-and-choose secure computation in the online/offline and batch settings. In: Garay, J.A., Gennaro, R. (eds.) CRYPTO 2014, Part II. LNCS, vol. 8617, pp. 476-494. Springer, Heidelberg (2014)

18. Malkhi, D., Nisan, N., Pinkas, B., Sella, Y.: Fairplay - a secure two-party computation system. In: Blaze, M. (ed.) 13th USENIX Security Symposium. USENIX Association (August 2004)

19. Mohassel, P., Franklin, M.: Efficiency tradeoffs for malicious two-party computation. In: Yung, M., Dodis, Y., Kiayias, A., Malkin, T. (eds.) PKC 2006. LNCS, vol. 3958, pp. 458-473. Springer, Heidelberg (2006)

20. Mohassel, P., Riva, B.: Garbled circuits checking garbled circuits: More efficient and secure two-party computation. In: Canetti, R., Garay, J.A. (eds.) CRYPTO 2013, Part II. LNCS, vol. 8043, pp. 36-53. Springer, Heidelberg (2013)

21. Nielsen, J.B., Nordholt, P.S., Orlandi, C., Burra, S.S.: A new approach to practical active-secure two-party computation. In: Safavi-Naini, R., Canetti, R. (eds.) CRYPTO 2012. LNCS, vol. 7417, pp. 681-700. Springer, Heidelberg (2012)

22. Nielsen, J.B., Orlandi, C.: LEGO for two-party secure computation. In: Reingold, O. (ed.) TCC 2009. LNCS, vol. 5444, pp. 368-386. Springer, Heidelberg (2009)

23. Pappas, V., Vo, B., Krell, F., Choi, S.G., Kolesnikov, V., Bellovin, S., Keromytis, A., Malkin, T.: Blind seer: A scalable private DBMS. In: 2014 IEEE Symposium on Security and Privacy. IEEE Computer Society Press (May 2014)

24. Pinkas, B.: Fair secure two-party computation. In: Biham, E. (ed.) EUROCRYPT 2003. LNCS, vol. 2656, pp. 87-105. Springer, Heidelberg (2003)

25. shelat, A., Shen, C.-h.: Two-Output Secure Computation with Malicious Adversaries. In: Paterson, K.G. (ed.) EUROCRYPT 2011. LNCS, vol. 6632, pp. 386-405. Springer, Heidelberg (2011)

26. Woodruff, D.P.: Revisiting the efficiency of malicious two-party computation. In: Naor, M. (ed.) EUROCRYPT 2007. LNCS, vol. 4515, pp. 79-96. Springer, Heidelberg (2007)

27. Yao, A.C.C.: How to generate and exchange secrets (extended abstract). In: 27th FOCS, pp. 162-167. IEEE Computer Society Press (October 1986) 